\title{
Accréditation en toxicologie
}

\section{Accreditation for toxicological laboratories}

\section{Anne GRUSON**(1), Gilbert PÉPIN ${ }^{(2)}$, Pierre MARQUET ${ }^{(3)}$, Jean-Pierre GOULLÉ(4), Michel LHERMITTE ${ }^{(\text {() }}$}

(1) Laboratoire de Biochimie-Toxicologie, Centre Hospitalier, 57, Avenue Winston Churchill - 62022 ARRAS Cedex Tél : 0321244528 - Fax : 0321244514 - E-mail : anne.gruson@ch-arras.fr

(2) Laboratoire TOXLAB, 7, rue Jacques Cartier - 75018 PARIS

Tél : 0158892800 - Fax : 0158592801 -E-mail : toxlab@wanadoo.fr

(3) Laboratoire de Pharmacologie et Toxicologie, CHU, 2, Avenue Martin Luther King - 87042 LIMOGES Cedex

Tél : 0555056140 - Fax : 0555056162 - E-mail : marquet@unilim.fr

(4) Laboratoire de Pharmacologie et de Toxicologie Cliniques, Groupe hospitalier - BP 24 76083 LE HAVRE Cedex - Tél : 0232733223 - Fax : 0232733238 - E-mail : jgoulle@ch-havre.fr (5) Laboratoire de Biochimie, Hôpital Calmette, Rue du Pr Leclercq - 59037 LILLE Cedex Tél : 0327714646 - Fax : 0327714647 -E-mail : mlhermitte@chru-lille.fr

** Tirés à part : Anne GRUSON

(Reçu le 12 décembre 2002 ; accepté le 22 décembre 2002)

\section{Introduction}

En France, l'organisme national reconnu par les instances européennes pour délivrer l'accréditation dans le contexte européen est le Comité Français d'Accréditation (COFRAC). La Commission Technique d'Accréditation «Santé» (biologie médicale, dispositifs médicaux et produits bio actifs) est l'une des 7 Commissions Techniques d'Accréditation du COFRAC. Au sein de celle-ci, un groupe à l'initiative de la commission «accréditation» de la Société Française de Toxicologie Analytique (SFTA) a rédigé un programme d'accréditation ayant pour domaine d'application la toxicologie et le suivi thérapeutique pharmacologique. Après avoir rappelé quelques notions sur le système d'assurance qualité (SAQ), les éléments nécessaires à l'accréditation dans le domaine des analyses de toxicologie seront détaillés, la liste des analyses permettant l'accréditation sera présentée. Les trente huit fiches correspondantes aux analytes ont été rédigées par des membres de la SFTA (Laboratoire de Biochimie et de Toxicologie, CHU de Poitiers, 350, Avenue Jacques Cœur 86021 Poitiers). Elles sont publiées sur le site Internet de la SFTA (www.SFTA.asso.fr). La fiche phénobarbital-thiopental sera présentée en exemple.

\section{Accréditation dans le contexte Européen et International \\ Le management de la qualité}


Le management de la qualité nécessite un Système d'Assurance Qualité (SAQ). Il impose au préalable de définir les objectifs de qualité, puis de préparer, de planifier et de réaliser les actions correspondantes. L'évaluation du SAQ est réalisée à l'aide d'audits qui examinent chaque activité. Ces audits sont importants lors de la mise en place de la démarche et pour sa pérennisation. La reconnaissance officielle d'un SAQ ne peut être accordée que par un organisme faisant autorité qui donne un "label" de type certification ou d'accréditation. L'accréditation atteste de l'existence d'un SAQ efficace et démontre en plus de la compétence et de la maitrise totale du processus technique.

Un certain nombre de laboratoires (privés, au début, mais aussi maintenant hospitaliers) ont réalisé, sont en cours ou s'engagent actuellement dans une démarche d'accréditation avec un référentiel s'inscrivant dans le contexte européen et international. L'accréditation est une démarche volontaire

L'"European Cooperation for Accreditation" ou "EA" a ainsi été mise en place par l'Union Européenne.

L'EA a pour objectif d'harmoniser les pratiques de l'accréditation dans les pays européens et dans le contexte international. Pour cette raison, l'EA travaille en liens privilégiés avec des organismes internationaux comme 1" "International Laboratory Accreditation Conference" (ILAC), et 1" International Accreditation Forum" (IAF).

L'EA reconnaît par état membre un organisme ayant autorité pour accréditer. Ces organismes nationaux reconnus bénéficient entre eux de reconnaissance mutuelle et d'accords multilatéraux (MLA). En France, l'organisme national, reconnu par les instances européennes pour délivrer l'accréditation européenne, est le Comité Français d'Accréditation (COFRAC).

L'organisme national d'accréditation est soumis à des contrôles périodiques par l'EA sur ses différents types d'activité. Le COFRAC comptait avant 2001, 35 commissions sectorielles d'accréditation, la plus récente étant celle de biologie. Depuis 2001, le COFRAC comprend 7 Commissions Techniques d'Accréditation (CTA) dont la Commission Technique d'Accréditation en "Santé» inspirée de l'"health care" des instances européennes. La CTA «Santé» rassemble la biologie médicale, les dispositifs médicaux et les produits bio actifs.

Les CTA dont les membres sont composés de clients, donneurs d'ordre, et d'Experts techniques du domaine émettent des propositions d'accréditation soumises à la Direction générale du COFRAC (sous couvert du Comité de Section), selon une procédure par rapporteurs qui accentuent le professionnalisme, l'efficacité et l'impartialité. Les CTA sont assistées par des collèges d'experts techniques. Les CTA élaborent aussi un certain nombre de documents de type «programme d'accréditation", afin de préciser les exigences sectorielles, si besoin.

\section{Le référentiel d'accréditation}

Le référentiel d'accréditation européen et international est à l'heure actuelle la norme ISO/CEI 17025 (1), complétée en France par le document L202A du COFRAC. Ce référentiel comporte deux parties.

\section{Première partie :}

Elle concerne les prescriptions relatives au système de management de la qualité : 1 - déterminer a priori la politique de qualité, en tenant compte de l'environnement, 2 - établir des prévisions et 3 - adapter les moyens et les compétences.

Dans ce cadre, un manuel d'assurance qualité (MAQ) décrit le système qualité, à partir duquel sont élaborés les procédures, les modes opératoires, les fiches techniques, les différents formulaires, ainsi que la structure de la documentation utilisée.

Le système de management de la qualité est guidé par une politique et des procédures fondées sur une géstion dynamique de l'ensemble : traçabilité, enregistrement des dysfonctionnements (non-conformités) avec actions préventives ou correctives, réclamations clients (patients et prescripteurs), sélection et utilisation de services, équipements et consommables.

La maîtrise de ce management nécessite la planification d'audits internes qui permet de s'assurer de la qualité du laboratoire.

\section{Deuxième partie :}

Elle comporte les ressources et les exigences techniques. Celles-ci sont relatives au personnel, locaux et conditions environnementales, matériels, procédures préanalytiques, analytiques, postanalytiques, comptes rendus de résultats. Les moyens demandés à ce niveau dans les référentiels sont très sensiblement proches de ceux exigés dans le guide de bonne exécution des analyses (GBEA) $(2,3)$

Le GBEA, lui, est un texte de spécificité française, réglementaire, obligatoire, soumis à contrôle aléatọire par les services déconcentrés de l'état : Direction Département de l'Action Sanitaire et Sociale (DDASS), Direction Régionale des Affaires Sanitaires et Sociales (DRASS). Le GBEA dans ses versions successives semblait s'orienter vers un management de la qualité en imposant dès les premières lignes du chapitre relatif aux "règles de fonctionnement" un "système d'assurance qualité". Cependant, l'aide à la mise en application attendue ensuite, n'est que partiellement développée. Le chapitre "assurance qualité" se posi- 
tionne toujours à la fin du document, et non au début, et n'annonce pas clairement une politique qualité (SAQ), avec manuel d'assurance qualité (MAQ), et reste toujours centré sur le contrôle de qualité.

La démarche du GBEA dans sa seconde version est donc intéressante avec une exigence forte en moyens, mais ne donnant que pour partie les clefs de la mise en œuvre de ces moyens, c'est-à-dire du management de la qualité.

Le tableau I montre les différents référentiels s'appliquant à un laboratoire d'analyses.

\section{Accréditation et domaine d'application en toxicologie}

Le programme d'accréditation d' «Analyses en Toxicologie et Suivi thérapeutique Pharmacologique» (4) porte sur des analyses réalisées à partir de prélèvements humains. Des accréditations pourront être délivrées pour tout ou partie des analyses définies dans le tableau II. Le programme reflète l'état d'avancement des connaissances au jour de leur approbation. Il est destiné à être régulièrement actualisé pour prendre en

Tableau I : Les différences références normatives d'un laboratoire d'analyses en toxicologie. *N.R. : notion Non Référencée dans ce texte normatif.

\begin{tabular}{|c|c|c|c|c|}
\hline & $\begin{array}{l}\text { Texte réglementaire } \\
\text { obligatoire }\end{array}$ & \multicolumn{3}{|c|}{ Démarche volontaire } \\
\hline & Inspection & Certification & \multicolumn{2}{|c|}{ Accréditation } \\
\hline Référentiel & GBEA & ISO 9002 & ISO/CEI 17025 & $\begin{array}{l}\text { Programme "Analyses en } \\
\text { toxicologie et suivi } \\
\text { thérapeutique" }\end{array}$ \\
\hline Objectif & Contrôle réglementaire & $\begin{array}{l}\text { Reconnaissance } \\
\text { de la conformité }\end{array}$ & \multicolumn{2}{|c|}{ Reconnaissance de la compétence } \\
\hline \multicolumn{5}{|c|}{ Nature du « label » de qualité } \\
\hline Domaine & $\begin{array}{l}\text { Les laboratoires d'analyses } \\
\text { biologiques et médicales }\end{array}$ & \begin{tabular}{c|} 
Service \\
Processus \\
Laboratoires \\
\end{tabular} & \multicolumn{2}{|c|}{$\begin{array}{l}\text { Organisme de certification, d'inspections et } \\
\text { laboratoires }\end{array}$} \\
\hline Autorité & DRASS & $\begin{array}{c}\text { Un organisme } \\
\text { indépendant de } \\
\text { certification } \\
\text { (ex : BVQI) }\end{array}$ & \multicolumn{2}{|c|}{$\begin{array}{l}\text { Organisme d'accréditation } \\
\text { COFRAC en France }\end{array}$} \\
\hline Evaluation & Rapport d'inspection & \multicolumn{3}{|c|}{ Référentiel normatif - Audit d'évaluation (rapport d'audit) } \\
\hline Acte délivré & Le droit d'exercer & $\begin{array}{l}\text { Certification } \\
\text { ISO }\end{array}$ & \multicolumn{2}{|c|}{ Accréditation internationale } \\
\hline \multicolumn{5}{|c|}{ Prescriptions relatives aux techniques } \\
\hline Equipements & \multicolumn{4}{|c|}{ Maintenance et métrologie des équipements } \\
\hline Locaux & \begin{tabular}{|c|} 
Installations \\
Elimination des déchets \\
\end{tabular} & N.R: & \multicolumn{2}{|c|}{ Aménagement des locaux } \\
\hline Prélèvements & \begin{tabular}{|c|}
$\begin{array}{c}\text { Prélèvement, identification et } \\
\text { conservation des échantillons } \\
\text { biologiques }\end{array}$ \\
\end{tabular} & $\begin{array}{c}\text { Identification } \\
\text { des échantillons } \\
\text { biologiques }\end{array}$ & \multicolumn{2}{|c|}{$\begin{array}{l}\text { Conditions de prélèvement, prélèvement, } \\
\text { identification et conservation des échantillons } \\
\text { biologiques, stockage prélèvements en plus }\end{array}$} \\
\hline Réactifs & $\begin{array}{l}\text { Stockage et utilisation des } \\
\text { matériels, réactifs industriels } \\
\text { et réactifs reconstitués }\end{array}$ & $\begin{array}{l}\text { Stockage des } \\
\text { réactifs }\end{array}$ & \multicolumn{2}{|c|}{$\begin{array}{l}\text { Stockage et utilisation des matériels, réactifs } \\
\text { industriels et réactifs reconstitués }\end{array}$} \\
\hline Contrôles & $\begin{array}{c}\text { Contrôles qualité internes et } \\
\text { externes }\end{array}$ & $\begin{array}{c}\text { Contrôles } \\
\text { internes }\end{array}$ & \multicolumn{2}{|c|}{ Contrôles qualité internes et extemes } \\
\hline \multirow{2}{*}{$\begin{array}{l}\text { Méthodes } \\
\text { d'analyses }\end{array}$} & \multicolumn{4}{|c|}{ Application des méthodes d'analyses choix des méthodes (ISO/CEI 17025) } \\
\hline & N.R. & \begin{tabular}{|c|} 
N.R. \\
\end{tabular} & \multicolumn{2}{|c|}{ Validation des méthodes } \\
\hline Résultats d'analyses & Comptes-rendus d'analyses & N.R. & $\begin{array}{c}\text { Rapport complet des } \\
\text { résultats }\end{array}$ & N.R. \\
\hline \multicolumn{5}{|c|}{ Prescriptions relatives a l'organisation du laboratoire } \\
\hline Personnel & $\begin{array}{l}\text { Organisation } \\
\text { Responsabilité de la } \\
\text { personne chargée de } \\
\text { l'assurance qualité }\end{array}$ & \multicolumn{2}{|c|}{$\begin{array}{c}\text { Organisation du personnel du } \\
\text { laboratoire (organigramme, compétence } \\
\text { du personnel, formation) responsabilité } \\
\text { de la direction, réunion d'évaluation du } \\
\text { système qualité }\end{array}$} & N.R. \\
\hline $\begin{array}{l}\text { Maîtrise des } \\
\text { documents et des } \\
\text { cnregistrements }\end{array}$ & $\begin{array}{l}\text { Procédures et modes } \\
\text { opératoires } \\
\text { Maitrise de l'informatique } \\
\text { Conservation des archives }\end{array}$ & \multicolumn{2}{|c|}{$\begin{array}{c}\text { Gestion des documents qualité } \\
\text { (procédure, mode opératoire) - } \\
\text { conservation des enregistrements tels } \\
\text { que le guideline }\end{array}$} & N.R. \\
\hline $\begin{array}{l}\text { Actions correctives } \\
\text { et préventives } \\
\text { Réclamations }\end{array}$ & N.R. & \multicolumn{2}{|c|}{$\begin{array}{c}\text { Audits internes } \\
\text { Moyens mis en cuuvre pour éviter les } \\
\text { non-conformités (amélioration du } \\
\text { système qualité) Réclamation des } \\
\text { clients (dossiers en retard...) }\end{array}$} & N.R. \\
\hline Evaluation & N.R. & \multicolumn{3}{|c|}{ Réalisation d'audit = vérification de la conformité des activités } \\
\hline Achat & N.R. & \multicolumn{2}{|c|}{$\begin{array}{l}\text { Achat du matériel et des fournitures } \\
\text { évaluation des foumisseurs, } \\
\text { responsable des commandes, étapes de } \\
\text { la facturation }\end{array}$} & N.R. \\
\hline Sous-traitance & N.R. & \multicolumn{3}{|c|}{ Evaluation des sous-traitants + " sous traitant accrédités » } \\
\hline
\end{tabular}


Tableau II : Code et liste des analytes. (d'après 4, Programme d'accréditation COFRAC n 168-01).

\begin{tabular}{|c|c|c|}
\hline CODES & ANALYTES & FICHES TECHNIQUES SFTA \\
\hline TOX. 10 & Amphétamines & Site : www.sfta.asso.fr \\
\hline TOX. 20 & Anticholinestérasigue & \\
\hline TOX. 30 & Benzodiazépines & \\
\hline TOX. 40 & Cannabis & \\
\hline TOX. 50 & Cocaïne & \\
\hline TOX. 60 & Cyanures & \\
\hline TOX. 70 & Digoxine - Digitoxine & \\
\hline TOX. 80 & Ethanol & \\
\hline TOX. 90 & Imipramine - Clomipramine & \\
\hline TOX. 100 & Lithium & \\
\hline TOX. 110 & Méprobamate & \\
\hline TOX. 120 & Methémoglobine & \\
\hline TOX. 130 & Opiacés & \\
\hline TOX. 140 & Oxyde de carbone & \\
\hline TOX. 150 & Paracétamol & \\
\hline TOX. 160 & Phénobarbital - Thiopental & \\
\hline TOX. 170 & Salicylés & \\
\hline TOX. 180 & Solvants chlorés & \\
\hline TOX. 190 & Antidépresseurs non Imipramique - non IMAO & \\
\hline TOX. 200 & Antipaludéens & \\
\hline TOX. 210 & Anti Vitamines K & \\
\hline TOX. 220 & Buprénorphine & \\
\hline TOX. 230 & Colchicine & \\
\hline TOX. 240 & Diurétiques & \\
\hline TOX. 250 & Insuline - Peptide C & \\
\hline TOX. 260 & LSD & \\
\hline TOX. 270 & Méthanol et Ethylène glycol & \\
\hline TOX. 280 & Méthadone & \\
\hline TOX. 290 & Neuroleptiques (Halopéridol-Cyamémazine - Sulpirine) & \\
\hline TOX. 300 & Organophosphorés & \\
\hline TOX. 310 & Paraquat, Diquat & \\
\hline TOX. 320 & Sulfhémoglobine & \\
\hline TOX. 330 & Aluminium & \\
\hline TOX. 340 & Arsenic & \\
\hline TOX. 350 & Cadmium, Cuivre, Zinc & \\
\hline TOX. 360 & Mercure & \\
\hline TOX. 370 & Plomb & \\
\hline TOX. 380 & Strontium & \\
\hline
\end{tabular}

compte l'évolution des technologies.

\section{Tableau des analytes - fiches techniques}

Les différents analytes (Tableau II) font l'objet de fiches techniques qui spécifient des principes et critères analytiques sur lesquels les laboratoires candidats à l'accréditation peuvent s'appuyer pour établir leurs protocoles opératoires.
Les fiches techniques sont publiées sur le site de la SFTA (www.sfta.asso.fr)

Chaque fiche a été rédigée selon un modèle unique et selon la demande particulière, analyse réalisée dans le cadre de la toxicologie clinique ou dans un cadre médico-légal.

Analyses toxicologiques par méthodes spécifiques 
Phase préanalytique :

- prélèvements (nature, quantité, nature du contenant, date et heure de prélèvement, mode de conservation des échantillons, date et heure de réception des échantillons au laboratoire, date et heure de l'analyse).

- Circonstances de l'intoxication

- Traitements connus (thérapeutique ou mise en œuvre pour traiter l'intoxication)

Phase analytique : identification, dosage par méthode spécifique

- les critères à remplir pour rechercher et doser le ou les xénobiotiques, aucune technique n'est imposée. Un travail bibliographique a permis de citer les méthodes les plus généralement utilisées, toutefois sont indiquées les limites de détection à atteindre, l'existence éventuelle d'interférences analytiques et surtout les pièges analytiques à éviter.

- Les données pharmacologique et toxicologique du xénobiotique sont rappelées : demi-vie, concentrations thérapeutiques, toxiques et létales, commentaires quant au résultat global : potentialisation, interaction,..

Phase postanalytique : conservation des échantillons

Lorsque les xénobiotiques peuvent être recherchés et dosés par méthode immunologique, celle-ci est indiquée, avec rappel des réactions croisées et des interférences possibles.

A titre d'exemple, deux fiches phénobarbital-thiopental en toxicologie hospitalière et en toxicologie médicolégale sont jointes en annexe. En toxicologie médicolégale, les limites de détection et de quantification sont données à titre indicatif. En effet, elles peuvent varier du fait que l'on ne maîtrise pas la qualité des prélèvements d'autopsie que l'on analyse. De même pour la toxicologie médico-légale, la validation ne concerne que le sang total et éventuellement le sérum et le plasma seuls milieux pour lesquels existent des données bibliographiques concernant les taux thérapeutiques, toxique's et mortels.

\section{Exigences}

L'aptitude d'un laboratoire à être accrédité par la Section Laboratoires du COFRAC est examinée au regard : 1 - des exigences générales du COFRAC, 2 des exigences techniques complémentaires spécifiques à une analyse ou à une catégorie d'analyses et précisées dans le présent programme, et 3 - de la jurisprudence du secteur de la biologie médicale qui apporte des précisions sur deux points explicités dans les référentiels précités : la définition du client d'un laboratoire de biologie médicale et la sous-traitance occasionnelle des analyses dans le cadre de l'accréditation. Ces points de jurisprudence ont été actés par la Commission
Permanente d'Accréditation en Biologie antérieure, et, repris dans tous les programmes gérés par la CTA «Santé».

Les exigences générales sont définies par la norme internationale NF EN ISO/CEI 17025 (4) et complétées par le document L202A du COFRAC (5). Il est rappelé que l'ISO (Organisation Internationale de Normalisation) et la CEI (Commission Electrotechnique Internationale) forment le système spécialisé de la normalisation mondiale. Les organismes nationaux membres de l'ISO ou de la CEI participent aux développements des normes internationales par l'intermédiaire des comités techniques créés par l'organisation concernée afin de s'occuper des domaines particuliers de l'activité technique. Les comités techniques de l'ISO et de la CEI collaborent dans des domaines d'intérêt commun.

Les exigences techniques spécifiques comprennent plusieurs points portant sur :

\section{1) - L' échantillonnage et la phase préanalytique}

Les échantillons doivent parvenir au laboratoire en quantité suffisante, dans des conditions qui évitent une modification de leurs caractéristiques chimiques et immunologiques. Si les échantillons ne satisfont manifestement pas à ces exigences, le laboratoire pourra, soit refuser les échantillons, soit émettre des réserves sur la signification des résultats.

Dans le cadre de la toxicologie médico-légale, les prélèvements sont effectués en respectant les prescriptions des consensus. Pour rappel, les prélèvements sont réalisés sous la responsabilité du médecin légiste ou d'une personne du corps médical habilitée à les pratiquer selon le consensus de la SFTA 1998 (6) et selon les recommandations du Conseil de l'Europe (7).

Dans le cadre des analyses de toxicologie clinique, les prélèvements sont effectués conformément aux exigences spécifiées dans les fiches techniques.

La nature du contenant doit être aréactive, afin d'éviter toute adsorption des xénobiotiques sur les parois et le relargage des constituants du contenant.

Le mode de conservation (pendant le transport et au laboratoire), est indiqué dans les fiches techniques.

Le délai de réalisation des analyses est spécifié dans les fiches techniques. Si aucun délai n'est indiqué, le laboratoire doit justifier son choix et apporter la preuve de la validité des résultats.

\section{2) - la phase analytique et la validation des méthodes}

Le laboratoire accrédité ou candidat à une accréditation au titre du présent programme, doit indiquer, pour chaque analyte, quelle méthode il emploie ou emploiera dans le cadre de son accréditation et apporter la preu- 
ve de sa validité. Les méthodes normalisées ou publiées ne doivent pas être considérées comme acquises et leur mise en application dans le laboratoire doit être validée. Dans le cadre de l'étude de recevabilité des demandes d'accréditation au titre du présent programme, le document 1133 (8) est applicable.

Le laboratoire devra toujours indiquer les outils de validation des méthodes d'analyses utilisées.

La validation de la qualité des prestations analytiques doit être réalisée par le contrôle de qualité interne obligatoire et d'autres contrôles d'évaluation externe de la qualité proposés par des sociétés organisatrices de tels contrôles.

Il est rappelé que, dans le cadre de l'accréditation, et sauf indication contraire de l'annexe technique du laboratoire, le COFRAC doit être averti préalablement à toute modification d'une méthode d'analyse $(9,10)$.

\section{3) - La phase analytique : matériels et réactifs}

Le matériel indispensable au fonctionnement des appareils doit être conforme aux prescriptions spécifiées par les constructeurs et être utilisé uniquement selon l'usage et les modalités prévus. La maintenance des appareils et équipements doit être assurée conformément aux spécifications du constructeur ou du fournisseur, en tenant compte des taches spécifiques dévolues soit au personnel du laboratoire, soit au service après vente du constructeur.

Les réactifs préparés ou reconstitués au laboratoire doivent porter la date de leur préparation ou reconstitution et celle de leur péremption; ceux d'origine industrielle doivent comporter, en outre, la date de leur réception au laboratoire et la date d'ouverture. Les instructions précises sur leurs conditions de stockage doivent être respectées.

Le laboratoire doit en particulier apporter la preuve que la température de chaque enceinte thermique est constamment maintenue dans un intervalle de valeurs correspondant aux exigences ou aux recommandations des méthodes d'analyse décrites dans les fiches techniques. Pour ce faire, le laboratoire doit avoir décrit et doit appliquer une procédure de mesure et d'enregistrement journalier des températures. Toutefois, pour les enceintes thermiques utilisées par intermittence, un enregistrement doit être effectué avant et en cours d'utilisation.

Les réactifs présentant un caractère toxique et/ou potentiellement contaminant doivent être stockés dans des conditions conformes à la réglementation en vigueur, et correctement étiquetés. Le personnel doit être instruit des particularités de stockage, d'élimination, et des mesures à prendre pour éviter tout risque en cas d'incident.
Les laboratoires de biologie médicale font un usage important des kits et systèmes commerciaux. Toutes les méthodes en biologie médicale utilisent des réactifs ayant fait l'objet d'un marquage CE (Communauté Européenne) (11) ou d'un enregistrement à l'AFSSAPS (Agence Française de Sécurité Sanitaire des Produits de Santé), suite à une expertise des dossiers fournisseurs, jusqu'à la fin de la période transitoire. Ces dossiers reposent sur des références bibliographiques et les critères fondamentaux de validation (linéarité, spécificité, sélectivité, limite de détection) sont en principe déterminés par le fabricant.

$\mathrm{Si}$ ces coffrets et systèmes sont utilisés sensu stricto dans les conditions préconisées par le fournisseur, les méthodes qui y font appel peuvent être considérées comme équivalentes a des méthodes normalisées. Il faut cependant noter que les analyseurs ne font pas l'objet d'un enregistrement AFSSAPS. Le couple analyseur-réactif doit en conséquence faire l'objet d'une évaluation dans l'environnement du laboratoire de toxicologie.

L'automate doit être considéré dans son ensemble (paramètres préanalytiques concernant l'appareil ou les échantillons qui lui sont soumis, facteurs analytiques incluant les méthodes analytiques, les températures de réaction, les mesures de temps et de densité optique etc..., les facteurs postanalytiques enfin permettant in fine de réaliser la validation technique des résultats).

\section{4) - La phase analytique : vérification des moyens d'analyses automatisés}

La vérification des moyens d'analyses automatisés doit être précisée. Elle doit être distincte de la validation des méthodes et de la métrologie, elle doit comprendre l'examen des contrats de maintenance souscrits auprès du fabricant et inclure les modalités de calibration, de contrôles et de validation technique et biologique. La validation des méthodes est une autre notion qu'il convient d'analyser différemment selon que l'on aura à faire à un laboratoire généraliste travaillant exclusivement sur des automates avec des techniques et des réactifs validés par le fabricant ou un laboratoire utilisant des méthodes ou réactifs "maison".

Ainsi, pour démontrer leur conformité au $\S 5-6$ du document L202A en ce qui concerne les automates, les laboratoires devront :

- valider les méthodes d'analyse en considérant le couple analyseur-réactifs dans leur environnement propre en évaluant les performances de la technique mise en œuvre et en vérifiant la conformité aux limites d'acceptabilité fixées. Cette validation s'appuiera sur des références scientifiques issues de la littérature et sur les données fournies par le constructeur ; 
- assurer la maintenance conformément aux spécifications du constructeur ou du fournisseur, en tenant compte des tâches spécifiques dévolues soit au personnel du laboratoire, soit au service après-vente du constructeur ;

- valider la qualité des prestations analytiques par le Contrôle de Qualité Interne obligatoire et d'autres contrôles d'Evaluation Externe de la Qualité proposés par des sociétés organisatrices de tels contrôles.

Les contrôles de qualité seront considérés comme une manière d'assurer la maîtrise des automates.

\section{5) - La phase analytique : métrologie des équipements de mesure et des moyens d'analyse (hors automate d'analyse)}

La traçabilité peut être assurée de deux manières :

- le laboratoire réalise lui même la vérification ou l'étalonnage de ses moyens de mesure ou d'analyse. Les dispositions prises par le laboratoire seront vérifiées par les auditeurs.

- le laboratoire sous-traite la vérification ou l'étalonnage de ses moyens à un laboratoire accrédité pour ses activités d'étalonnage et de vérification par le COFRAC ou par un organisme signataire de l'accord multilatéral EA.

En ce qui concerne la métrologie, l'absence ou un défaut de traçabilité des moyens de mesure ou d'analyse ayant une incidence sur les résultats des analyses, est un écart au référentiel d'accréditation qui sera classé en non-conformité.

\section{6) - La phase analytique : les locaux}

Le laboratoire doit apporter la preuve que les analyses se déroulent dans des conditions d'ambiance des locaux (température, exposition au soleil ...) conformes aux exigences des méthodes d'analyse, et à la bonne conservation des échantillons.

\section{7) - La phase analytique : les produits soumis à l'ana- lyse}

Le laboratoire doit mettre en place des dispositions qui assurent la traçabilité des échantillons tout au long du processus de traitement et d'analyse, y compris lors des étapes au cours desquelles les échantillons ne sont identifiables que d'après le repère de leur position dans l'espace et non par marquage individuel.

\section{8) - La phase postanalytique}

Le mode de conservation après analyse doit être précisé : les cheveux à température ambiante et au sec, les autres prélèvements biologiques, soit réfrigérés $(0$ à $\left.8^{\circ} \mathrm{C}\right)$, soit congelés $\left(<-18^{\circ} \mathrm{C}\right)$, sauf indication contraire mentionnée dans la fiche technique correspondante.

Les produits soumis à analyse doivent être éliminés selon des modalités qui évitent toute contamination.

Le délai de conservation après analyse en toxicologie clinique doit être au minimum de 7 jours.

En toxicologie médico-légale, le délai de conservation des scellés est :

- soit un minimum imposé par la loi (fixé à 9 mois pour un flacon de contre-expertise d'alcoolémie dans le cadre de la circulation routière et des crimes et délits de droit commun),

- soit jusqu'à l'accord de destruction par le parquet ou le magistrat instructeur.

Le laboratoire doit mettre en place des dispositions qui assurent la conservation des échantillons jusqu'à leur élimination ou réutilisation. Les scellés ne pourront être détruits par le laboratoire que selon les modalités agréées par les autorités judiciaires.

\section{Évaluation}

Réalisation des audits : selon le type d'audit, l'équipe chargée des opérations d'évaluation est composée d'un auditeur qualiticien et d'un ou plusieurs experts techniques spécialistes des analyses objet du présent programme, ou d'un auditeur technique formé à la qualité, spécialiste de ces mêmes analyses. Les laboratoires doivent être en activité lors de l'audit. L'audit technique s'appuiera sur les fiches techniques référencées dans les tableaux du présent programme.

Les laboratoires accrédités au titre du programme «Analyses en Toxicologie et Suivi Thérapeutique Pharmacologique» (4) s'engagent à participer à leurs frais aux essais interlaboratoires dont la fréquence et la nature sont décidées par la Commission Technique d'Accréditation «Santé». Ils doivent utiliser, lorsqu'ils sont disponibles, des matériaux de référence certifiés (MRC), soit par un organisme accrédité par le COFRAC, soit tout autre disposition apportant des garanties équivalentes de leur qualité.

\section{La démarche d'accrédita- tion n'est pas figée}

La démarche de qualité est permanente et continue. Ainsi, le référentiel européen et les normes ISO évoluent. La norme ISO/CEI 17025 devrait intégrer également l'évolution 2000 des ISO 9000 . Elle est donc particulièrement bien adaptée au management d'un système qualité de laboratoire, de type certification, en incluant aussi la compétence technique.

Parallèlement il existe un projet de norme ISO/DIS 15189. Il est relatif aux "Exigences particulières concernant la qualité et la compétence" pour les 
Laboratoires d'Analyses de Biologie Médicale. Cette norme inclut aussi la notion de compétence et pourrait être utilisée par les organismes d'accréditation comme le COFRAC comme norme pour l'accréditation. Elle présente en annexe la correspondance avec L'ISO/CEI 17025 dont elle respecte l'ensemble des principes généraux.

La validation des techniques (demandée aux laboratoires accrédités ou candidats à l'accréditation en Biologie médicale) s'inscrit dans le contexte de l'application de la directive européenne aux réactifs de laboratoire. Les différents documents GBEA, Normes ISO 17025 et 15189 montrent combien cette validation est d'actualité dans le nouveau contexte européen.

\section{Conclusion}

Les travaux du groupe émanant de la Commission «Accréditation» SFTA ont donc permis l'élaboration du programme d'accréditation «toxicologie» par le COFRAC qui positionne cette discipline au même titre que les autres domaines biologiques. Des experts techniques sont actuellement recrutés pour conduire des audits d'évaluation sur ce programme. Les laboratoires candidats à l'accréditation peuvent dès à présent engager leur démarche.

\section{Remerciements}

Ils s'adressent à tous les :

- membres du groupe «Toxicologie et Suivi Thérapeutique» du Cofrac : Allio I., Alvarez JC, Arditti J, Billaud E, Bouquet S, Capolaghi B, Compagnon P, Delhotal B, Deveaux M, Dreyfuss MF, Dupont A, Goullé JP, Gruson A, Houin G, Lacarelle B, Lhermitte M, Marquet P, Molinaro R, Nerot-Morin S, Pepin G, Perrin M, Peytavin G, Plesse J, Royer-Morrot MJ, Thevenot MT,

- auteurs ayant également participé à l'élaboration et à la relecture des fiches de toxicologie : Allio I, Alvarez JC, Anger JP, Arditti J, Baruthio T, Billaud E, Capolaghi B, Cheze M, Compagnon P, Dehon B, Deveaux M, Dreyfuss MF, Dubourvieux N, DumestreToulet V, Gaillard Y, Ghysel MH, Goullé JP, Gruson A, Houdret N, Houin G, Kintz P, Lacassie E, Lachâtre G, Lafargue P, Le Bouil A, Lhermitte M, Marka C, Marquet P, Molinaro R, Mura P, Pépin G, Pineau A, Plesse J, Ragoucy-Sengler C, Ricordel I, Royer-Morot MJ, Thevenot MT, Tourneau J, Tracqui A, Turcant A, Vestraet A, Vincent F, Vinner E.

\section{Références}

1. NF EN ISO/CEI 17025 : «Prescriptions générales concernant la compétence des laboratoires d'étalonnage et d'essais», (mai 2000) AFNOR (www.afnor.fr).

2. Arrêté du 2 novembre 1994 relatif à la bonne exécution des analyses de biologie médicale (GBEA).

3. Arrêté du 29 Novembre 1999 relatif à la bonne exécution des analyses de biologie médicale (GBEA).

4. Programme d'accréditation «Analyses en toxicologie et suivi thérapeutique pharmacologique», $\mathrm{n}^{\circ}$ 168-01 (révision 1 - septembre 2002) COFRAC (www.cofrac.fr).

5. Document L202A «Guide d'utilisation de la norme NF EN ISO/CEI 17025 à l'usage des laboratoires accrédités ou candidats à l'accréditation» [révision 01-février 2001], COFRAC(www.cofrac.fr).

6. Pépin G., Deveaux M., Goullé J.P., Kintz P., Marquet P. Les prélèvements d'autopsie nécessaires à la bonne exécution des expertises toxicologiques Toxicorama (1998), $\mathrm{X} ; 3: 110-119$.

7. Conseil de l'Europe. Recommandations $n^{\circ} \mathrm{R}$ (99) 3 relative à l'harmonisation des règles en matière d'autopsie médico-légale. 1999.

8. Document 1133 : «Validation interne des méthodes d'essais» [révision 00 - octobre 1995], COFRAC (www.cofrac.fr).

9. Analyses de biologie médicale : spécification et normes d'acceptabilité à l'usage de la validation des techiques » Ann Biol Clin, 1999, 57, 685-95.

10. Procédure de validation d'une technique, Spectra Biol, 1997, 16, 90, 43-50.

11. Directive 98/79/CE du parlement européen et du Conseil du 27 octobre 1998 relative aux dispositifs médicaux de diagnostic in vitro, IO CE L 331 (07 décembre 1998). 


\section{Annexes}

\section{PHENOBARBITAL ET THIOPENTAL (toxicologie clinique)}

\section{Analyses toxicologiques par méthodes spécifiques}

\section{A. Phase préanalytique}

1. Prélèvements

1.1. Nature : sang, liquide de lavage gastrique, urines

1.2. Quantité : 0,5 à $2 \mathrm{ml}$ de liquide biologique

1.3. Nature du contenant : tube en verre

1.4.Date et heure du prélèvement:

1.5.Mode de conservation des échantillons :

souhaité : $+4^{\circ} \mathrm{C}$ pour les analyses programmées dans les $24 \mathrm{~h}$, à $-20^{\circ} \mathrm{C}$ pour les analyses différées.

réel :

1.6. Date et heure de réception de l'échantillon au laboratoire

\subsection{Date et heure d'analyse}

\section{Circonstances de l'intoxication}

2.1. Inconnue

2.2. Connue Les cas d'intoxication sont consécutifs à une tentative de suicide ou à un accident d'anesthésie. Certains cas sont accidentels chez l'enfant ou des toxicomanes.

L'intoxication par les barbituriques notamment ceux d'action rapide est une urgence extrême (arrêt respiratoire).

\section{Traitements}

\subsection{Thérapeutique du sujet} inconnue :

connue : nature, posologie, date et heure de la thérapeutique,

\subsection{Thérapeutique mise en œuvre dans le cadre de l' intoxication} inconnue

connue : nature, posologie ,date et heure de la thérapeutique (Diurèse osmotique alcaline)

\section{B. Phase analytique : identification, dosage par méthode spécifique}

\section{1- Critères à remplir pour la recherche et/ou le dosage}

\section{Méthodes utilisées :}

$>$ phénobarbital : le dosage est généralement réalisé par immunoanalyse (polarisation de fluorescence ou EMIT). Cette technique robuste permet la quantification du xénobiotique sur une plage de concentrations, incluant largement la zone thérapeutique. Des méthodes chromatographiques sont toutefois disponibles.

$>$ thiopental, deux types de méthodes chromatographiques peuvent être retenues:

- CLHP - UV : à polarité de phase inverse (colonne C18) avec une phase mobile acétonitrile-tampon, extraction par déprotéinisation à l'acétonitrile ou liquideliquide à $\mathrm{pH}$ acide

- CPG - SM : extraction en milieu acide [il existe du phénobarbital-D5 (phényl- $\mathrm{D}_{5}$ ) comme étalon interne]. 
1.1. Limite de quantification : polarisation de fluorescence $: 1,1 \mathrm{mg} / \mathrm{l}$

1.2. Validation de la méthode : selon les critères du laboratoire

1.3. Existence d'interférences analytiques : polarisation de fluorescence : les composés suivants ont provoqué moins de $10 \%$ d'erreur : bilirubine $150 \mathrm{mg} / \mathrm{l}$, hémoglobine $10 \mathrm{~g} / \mathrm{l}$, triglycérides $25 \mathrm{~g} / \mathrm{l}$, protéines 30 à $100 \mathrm{~g} / \mathrm{l}$.

1.4. Pièges à éviter : CPG et CPG-SM : en présence de traces d'agents de méthylation dans l'injecteur, le phénobarbital se méthyle facilement en méphobarbital.

\section{Données pharmacologiques et toxicologiques}

$>$ sang : le rapport des concentrations sang/plasma est voisin de 1 pour le phénobarbital.

$>$ le lavage gastrique : n'est plus recommandé que pour les toxiques lésionnels ou pour les toxiques fonctionnels à forte toxicité (consensus de la Société de Réanimation de Langue Française).

$>$ urine pour le phénobarbital dont une fraction (30\%) est éliminée par le rein sous forme inchangée, d'autant plus que les urines sont alcalines. Le thiopental est entièrement métabolisé au niveau hépatique (dont en pentobarbital).

Métabolisme hépatique par oxydation ou hydroxylation conduisant à des dérivés inactifs, éliminés dans les urines après glucuroconjugaison.

\subsection{Demi-vie :}

$>$ phénobarbital : $50-140 \mathrm{~h}$ chez l'adulte, $40-70 \mathrm{~h}$ chez l'enfant

Augmentation chez le sujet âgé ou en cas d'insuffisance hépatique ou rénale. Le pic plasmatique est obtenu respectivement 8 heures et 4 heures après l'ingestion chez l'adulte et chez l'enfant.

$>$ thiopental : $10 \mathrm{~h}$ environ

Augmentation chez le sujet âgé ou en cas d'insuffisance hépatique.

\subsection{Concentrations thérapeutiques, toxiques et létales}

\section{$>$ phénobarbital:}

- dosage toxique : $500 \mathrm{mg}$ chez l'adulte, $20 \mathrm{mg} / \mathrm{kg}$ chez l'enfant

- concentrations thérapeutiques : 15-40 mg/L chez l'adulte, $20-60 \mathrm{mg} / \mathrm{L}$ chez l'enfant

- concentrations toxiques : $>50 \mathrm{mg} / \mathrm{L}$

Il existe une relation entre les taux plasmatiques et l'intensité du coma :

\begin{tabular}{|l|l|l|}
\hline \multicolumn{3}{|l|}{ Relation phénobarbital sanguin et intensité du coma } \\
\hline & Sujet naïf & Induction hépatique aux barbituriques \\
\hline Coma 0 & $53 \pm 20$ & $78 \pm 36$ \\
\hline Coma I & $61 \pm 31$ & 121 \\
\hline Coma II & $68 \pm 34$ & 116 \\
\hline Coma III & $98 \pm 40$ & 160 \\
\hline Coma IV & $135 \pm 52$ & -- \\
\hline
\end{tabular}

\section{$>$ thiopental}

- dosage toxique : 0,5 - $1 \mathrm{~g}$ chez l'adulte, $20 \mathrm{mg} / \mathrm{kg}$ chez l'enfant

- concentrations thérapeutiques : variables en fonction de l'indication (valeurs sous ventilation assistée) :

- neurotraumatologie 60 à $100 \mathrm{mg} / \mathrm{l}$ (posologie 5 à $12 \mathrm{mg} / \mathrm{kg} / \mathrm{h}$ )

- contrôle états épileptiques 15 à $50 \mathrm{mg} / \mathrm{l}$

- contrôle hypertension intracrânienne 10 à $15 \mathrm{mg} / \mathrm{l}$ (posologie 1 à $4 \mathrm{mg} / \mathrm{kg} / \mathrm{j}$ )

- récupération d'une activité cérébrale : conc de 15 à $30 \mathrm{mg} / \mathrm{l}$ récupération d'une ventilation spontanée : conc $\simeq 7,5 \mathrm{mg} / \mathrm{l}$ 
- concentrations toxiques : $>7 \mathrm{mg} / \mathrm{l}$ en respiration spontanée

$>100 \mathrm{mg} / \mathrm{l}$ en ventilation assistée

- concentrations létales : 10-100 mg/l en respiration spontanée

\section{Commentaires quant au résultat global : potentialisation, interaction,..}

La dose létale de barbiturique varie en fonction de nombreux facteurs mais une intoxication sévère risque de se produire lors de l'ingestion d'une dose dix fois supérieure à la dose hypnotique. Si l'alcool ou d'autres dépresseurs du SNC sont associés, la concentration létale est plus faible.

Les barbituriques sont parfois associés à des principes actifs présentant une toxicité propre. Certains, comme la prométhazine, augmentent la durée d'action des barbituriques d'action rapide.

L'interprétation de la concentration en phénobarbital doit tenir compte de la tolérance du patient : épilepsie connue et traitée ou toxicomanie.

Les concentrations de phénobarbital sont augmentées de $40 \%$ lors d'association avec l'acide valproïque. (inhibition enzymatique)

\section{Phase post-analytique}

Mode de conservation après analyse : $-20^{\circ} \mathrm{C}$, délai selon GBEA

\section{Dépistage par méthode immunochimique}

La recherche qualitative, dans un but d'orientation toxicologique, peut être effectué dans les urines, le sérum ou le liquide de lavage gastrique. Elle met en œuvre des techniques d'immunodosage, qui à l'aide d'anticorps faiblement spécifiques (anticorps anti-sécobarbital dans les techniques FPIA et EMIT), reconnaissent de manière variable les différents barbituriques, sans ou avec peu d'interférence avec leurs métabolites hydroxylés. Dans les urines, le seuil de positivité préconisé par le National Institute fot Drugs of Abuse (NIDA) est établi à $200 \mathrm{ng} / \mathrm{ml}$.

Un dosage spécifique du phénobarbital est réalisé grâce à des kits de réactifs faisant intervenir des anticorps spécifiques anti-phénobarbital.

\section{BIBLIOGRAPHIE}

\section{Dictionnaire Vidal en vigueur}

Russo H., Bressolle F. Pharmacodynamics and pharmacokinetics of thiopental. Clin Pharmacokinet $1998 ; 35: 95-134$.

Bismuth C. et coll. Toxicologie clinique. Médecine-scicences Flammarion éditeur. Paris 1987. 


\section{PHENOBARBITAL ET THIOPENTAL}

(toxicologie médico-légale)

\section{Analyses toxicologiques par méthodes spécifiques}

\section{A. Phase préanalytique \\ 1. Prélèvements}

1.1 Nature : Sang périphérique, sang cardiaque, urınes, bile, contenu gastrique, cheveux, tissus (cerveau en particulier, cœur, tissu adipeux)

1.2. Quantité : $10 \mathrm{ml}$ de liquide biologique ou $10 \mathrm{~g}$ de viscères, sous la responsabilité du médecin légiste,

1.3. Nature du contenant : tube ou flacon en verre ou polypropylène.

1.4. Date et heure des faits :
a Inconnu
Connu :

\subsection{Date et heure du décès}

$\square$ Inconnu $\square$ Connu :

\subsection{Mode de conservation du corps}

$\square$ Inconnu $\square$ Connu :

\subsection{Date et heure du prélèvement}

$\square$ Inconnu $\square$ Connu :

1.8 Mode de conservation des échantillons

Souhaité : 0 à $8^{\circ} \mathrm{C}$ ou congelé $\left(<-18^{\circ} \mathrm{C}\right)$

réel

1.9. Date et heure de réception des échantillons au laboratoire

1.10. Date et heure des analyses

\section{Circonstances et causes de l'intoxication}

\section{Circonstances de l'intoxication}

2.1. Inconnue

\subsection{Connue}

Les cas d'intoxication sont consécutifs à une tentative de suicide ou à un accident d'anesthésie. Certains cas sont accidentels chez l'enfant ou des toxicomanes.

L'intoxication par les barbituriques notamment ceux d'action rapide est une urgence extrême (arrêt respiratoire).

\section{Cause de l'intoxication}

\section{Traitements}

\subsection{Thérapeutique du sujet}

inconnue

connue : nature, posologie, date et heure de la thérapeutique, 


\subsection{Thérapeutique mise en oeuvre dans le cadre de l' intoxication inconnue connue : nature, posologie ,date et heure de la thérapeutique(Diurèse osmotique alcaline)}

\section{B. Phase analytique : identification, dosage par méthode spécifique}

\section{Critères à remplir pour la recherche et/ou le dosage}

\section{Méthodes utilisées :}

Deux types de méthodes chromatographiques peuvent être retenues :

- CLHP - UV : à polarité de phase inverse (colonne C18) avec une phase mobile acétonitrile-tampon, extraction par déprotéinisation à l'acétonitrile ou liquideliquide à $\mathrm{pH}$ acide

- CPG - SM : extraction en milieu acide (il existe un phénobarbital-D5 comme étalon interne).

Les limites de détection et de quantification sont données à titre indicatif. En effet, elles peuvent varier du fait que l'on ne maîtrise pas la qualité des prélèvements d'autopsie que l'on analyse. La validation ne concerne que le sang total et éventuellement le sérum et le plasma seuls milieux pour lesquels existent des données bibliographiques concernant les taux thérapeutiques, toxiques et mortels.

\subsection{Limite de détection et/ou de quantification: polarisation de fluorescence : $1,1 \mathrm{mg} / \mathrm{l}$.}

1.2. Validation de la méthode : selon les critères du laboratoire

1.3. Existence d'interférences analytiques : polarisation de fluorescence : les composés suivants ont provoqué moins de $10 \%$ d'erreur : bilirubine $150 \mathrm{mg} / \mathrm{l}$, hémoglobine $10 \mathrm{~g} / \mathrm{l}$, triglycérides $25 \mathrm{~g} / \mathrm{l}$, protéines 30 à $100 \mathrm{~g} / \mathrm{l}$.

1.4. Pièges à éviter : $\mathrm{CPG}$ et CPG-SM : en présence de traces d'agents de méthylation dans l'injecteur, le phénobarbital se méthyle facilement en méthobarbital.

\section{Données pharmacologiques et toxicologiques}

sang : le rapport des concentrations sang/plasma est voisin de 1 pour le phénobarbital.

$>$ le lavage gastrique, n'est plus recommandé que pour les toxiques lésionnels ou pour les toxiques fonctionnels à forte toxicité (consensus de la Société de Réanimation de Langue Française).

$>$ tissus (cerveau en particulier, cœur, tissu adipeux) du fait d'une large diffusion dans l'organisme liée à une liposolubilité très importante. Les volumes de distribution sont respectivement de 0.54 et $2.3 \mathrm{l} / \mathrm{kg}$ pour le phénobarbital et le thiopental. Ce dernier subit une redistribution après injection IV au profit des tissus les moins vascularisés corrélée à une diminution des concentrations dans le plasma et le cerveau. Des administrations répétées peuvent induire une accumulation dans le tissu adipeux.

urine : pour le phénobarbital dont une fraction $(30 \%)$ est éliminée par le rein sous forme inchangée, d'autant plus que les urines sont alcalines. Le thiopental est entièrement métabolisé au niveau hépatique (dont en pentobarbital). 


\subsection{Demi-vie :}

$>$ phénobarbital : $50-140 \mathrm{~h}$ chez l'adulte, $40-70 \mathrm{~h}$ chez l'enfant

Augmentation chez le sujet âgé ou en cas d'insuffisance hépatique ou rénale. Le pic plasmatique est obtenu respectivement 8 heures et 4 heures après l'ingestion chez l'adulte et chez l'enfant.

$>$ thiopental : $10 \mathrm{~h}$ environ

Augmentation chez le sujet âgé ou en cas d'insuffisance hépatique.

\subsection{Concentrations thérapeutiques, toxiques et létales (du xénobiotique ou du métabolite)}

$>$ phénobarbital :

- dosage toxique : $500 \mathrm{mg}$ chez l'adulte, $20 \mathrm{mg} / \mathrm{kg}$ chez l'enfant

- concentrations thérapeutiques : $15-40 \mathrm{mg} / \mathrm{L}$ chez l'adulte, $20-60 \mathrm{mg} / \mathrm{L}$ chez l'enfant

- concentrations toxiques : $>50 \mathrm{mg} / \mathrm{L}$

Il existe une relation entre les taux plasmatiques et l'intensité du coma. Un coma stade IV est généralement observé au delà de $100 \mathrm{mg} / \mathrm{L}$.

$>$ thiopental

- dosage toxique : $0,5-1 \mathrm{~g}$ chez l'adulte, $20 \mathrm{mg} / \mathrm{kg}$ chez l'enfant

- concentrations thérapeutiques : variables en fonction de l'intoxication :

- neurotraumatologie 60 à $100 \mathrm{mg} / \mathrm{l}$ (posologie 5 à $12 \mathrm{mg} / \mathrm{kg} / \mathrm{h}$ )

- contrôle hypertension intracrânienne 10 à $15 \mathrm{mg} / \mathrm{l}$ (posologie 1 à $4 \mathrm{mg} / \mathrm{kg} / \mathrm{j}$ )

- récupération d'une activité cérébrale : conc de 15 à $30 \mathrm{mg} / \mathrm{l}$

- récupération d'une ventilation spontanée : conc $\simeq 7,5 \mathrm{mg} / \mathrm{l}$

- contrôle états épileptiques 15 à $50 \mathrm{mg} / \mathrm{l}$

- concentrations toxiques : > $7 \mathrm{mg} / \mathrm{l}$ en respiration spontanée $>100 \mathrm{mg} / \mathrm{l}$ en ventilation assistée

- concentrations létales : 10 à $100 \mathrm{mg} / \mathrm{l}$ en respiration spontanée (dans 2 cas de suicide, les concentrations suivantes ont été obtenues : 153 et $273 \mathrm{mg} / \mathrm{l}$ ).

La dose létale de barbiturique varie en fonction de nombreux facteurs mais une intoxication sévère risque de se produire lors de l'ingestion d'une dose dix fois supérieure à la dose hypnotique. Si l'alcool ou d'autres dépresseurs du SNC sont associés, la concentration létale est plus faible.

Métabolisme hépatique par oxydation ou hydroxylation conduisant à des dérivés inactifs, éliminés dans les urines après glucuroconjugaison

\subsection{Stabilité dans les milieux biologiques : non renseignée}

\section{Commentaires quant au résultat global : potentialisation, interaction,..}

Les barbituriques sont parfois associés à des principes actifs présentant une toxicité propre. Certains, comme la prométhazine, augmentent la durée d'action des barbituriques d'action rapide.

L'interprétation de la concentration en phénobarbital doit tenir compte de la tolérance du patient : épilepsie connue et traitée ou toxicomanie.

Les concentrations de phénobarbital sont augmentées de $40 \%$ lors d'association avec l'acide valprö̈que. (inhibition enzymatique)

\section{Phase post-analytique}

Mode de conservation après analyse : 0 à $8^{\circ} \mathrm{C}$ ou congélation $\left(<-18^{\circ} \mathrm{C}\right)$ 


\section{Dépistage par méthode immunochimique à confirmer obligatoirement par méthode spécifique}

La recherche qualitative, dans un but d'orientation toxicologique, peut être effectué dans les urines, le sérum ou le liquide de lavage gastrique. Elle met en œuvre des techniques d'immunodosage, qui à l'aide d'anticorps faiblement spécifiques (anticorps anti-sécobarbital dans les techniques FPIA et EMIT), reconnaissent de manière variable les différents barbituriques, sans ou avec peu d'interférence avec leurs métabolites hydroxylés. Dans les urines, le seuil de positivité préconisé par le National Institute fot Drugs of Abuse (NIDA) est établi à $200 \mathrm{ng} / \mathrm{ml}$.

Un dosage spécifique du phénobarbital est réalisé grâce à des kits de réactifs faisant intervenir des anticorps spécifiques anti-phénobarbital.

Réactions croisées si elles existent

\section{Bibliographie}

\section{Dictionnaire Vidal en vigueur}

G. Pépin et al. Les prélèvements d'autopsie nécessaires à la bonne exécution des expertises toxicologiques, Toxicorama, 1998 (3), 110-119.

Russo H., Bressolle F. Pharmacodynamics and pharmacokinetics of thiopental. Clin Pharmacokinet 1998 ; 35 : 95-134. 